\title{
A note on the semidiurnal non-migrating tide at polar latitudes
}

\author{
Takehiko Aso \\ National Institute of Polar Research, Research Organization of Information and Systems, Kaga 1-9-10, Itabashi, Tokyo 173-8515, Japan
}

(Received March 5, 2007; Revised June 7, 2007; Accepted June 11, 2007; Online published July 18, 2007)

\begin{abstract}
In the Antarctic upper mesosphere and lower thermosphere around $90 \mathrm{~km}$, meteor radar observations at the South Pole have detected a significant semidiurnal wind component in summer which is found to be nonmigrating with zonal wavenumber $s=1$. It has been surmised that this component might possibly be excited through the non-linear interaction of the migrating semidiurnal tide with stationary planetary waves with zonal wavenumber $s=1$ prevailing at stratospheric heights. The Kyushu University GCM has been successful in elucidating very unambiguously this conjecture. In the present paper, linearized steady tidal modeling is carried out in this connection to reproduce, by a fairly simplified but explicit model, trans-equatorial propagation of non-migrating semidiurnal tide forced in the opposite winter hemisphere and to compare latitudinal structures of migrating and non-linearly excited intermittent tides in view of polar latitudes where non-migrating tide tends to dominate over migrating tide. It is also shown that the re-analysis meteorological data for almost 10 years clearly supports the well-known N-S asymmetry in stationary planetary wave activity in winter polar stratospheric regions, possibly due to the difference in surface topography between two hemispheres. We suggest that there might be significant asymmetry in the summertime enhancement of semidiurnal non-migrating tide between both polar regions. This phenomenon may be in a context similar with the N-S asymmetry of gravity wave activity in the polar regions, possibly giving rise to $\mathrm{N}-\mathrm{S}$ disparity of cold summertime mesopause temperature. Clarification awaits intensive bi-polar studies by coordinated radar and optical observations which are running both in the Arctic and Antarctic regions.
\end{abstract}

Key words: Semidiurnal tide, non-migrating tide, stationary planetary wave, polar mesosphere and lower thermosphere.

\section{Introduction}

Semidiurnal 12-h oscillations are still a dominant atmospheric wave in the high-latitude mesosphere and lower thermosphere in that non-migrating (zonal wavenumber $s=1)$ or zonally symmetric $(s=0)$ tides have non-zero wind value near the poles. Hence it could be observed by radars as meteor and MF radars and optical observations like lidar and imager. Long-term studies have been made in view of its variability and connection to global dynamical couplings of vast atmospheric layers. It varies in fairly broad temporal scales according to various agents, such as forcing, background wind and latitudinal temperature gradient (Aso et al., 1981, 1987; Forbes, 1982a, b), and nonlinear interaction with other wave regimes (Miyahara, 1999). At higher latitudes, wind velocity components of migrating semidiurnal tide which has zonal wavenumber $s=2$ should tend to vanish as the wind components $180^{\circ}$ apart in longitude should be out of phase across the pole. Forbes et al. (1995) found that this is the case at the South Pole observation by steering a beam in 4 directions around the South Pole; the zonal wavenumber of the 12-h component was found to be one, i.e. it is of a non-migrating nature. They also found that the amplitude is significant only in summertime and that the semidiurnal component is fairly weak

Copy right (C) The Society of Geomagnetism and Earth, Planetary and Space Sciences (SGEPSS); The Seismological Society of Japan; The Volcanological Society of Japan; The Geodetic Society of Japan; The Japanese Society for Planetary Sciences; TERRAPUB and intermittent in winter (Portnyagin et al., 1998, Fig. 1; Forbes et al., 1999). They surmised that this non-migrating semidiurnal component is excited in terms of nonlinear interaction of the migrating $s=2$ semidiurnal tide with stationary planetary wave with $s=1$, which is excited in the troposphere and propagates into the winter stratosphere.

Yamashita et al. (2002) performed a comprehensive Kyushu University-GCM calculation and confirmed that the non-linear interaction with stationary planetary waves is really taking place, contributing to generation of the $s=1$ semidiurnal component. They also calculated EP fluxes and, together with the phase structure of this component, suggested that this is excited in the winter hemisphere and propagates across the equator up to mesosphere and to lower thermosphere heights in the summer hemisphere, giving rise to summertime enhancement at polar MLT regions.

In the following, a linearized and steady-state calculation of semidiurnal non-migrating tide is conducted using our ATM2 model (Aso et al., 1987) by assuming perpetual heating with $s=1$ to delineate how it propagates to meteor heights in a simplified model, This can also suggest-in a simplified and explicit way-how this non-migrating component behaves relative to migrating one at higher latitudes. Asymmetry of stationary planetary wave activity between two hemispheres is then illustrated with available long-term synoptic meteorological data. Discussions on the expected asymmetry in semidiurnal non-migrating tide at both polar regions are given in the last section. 

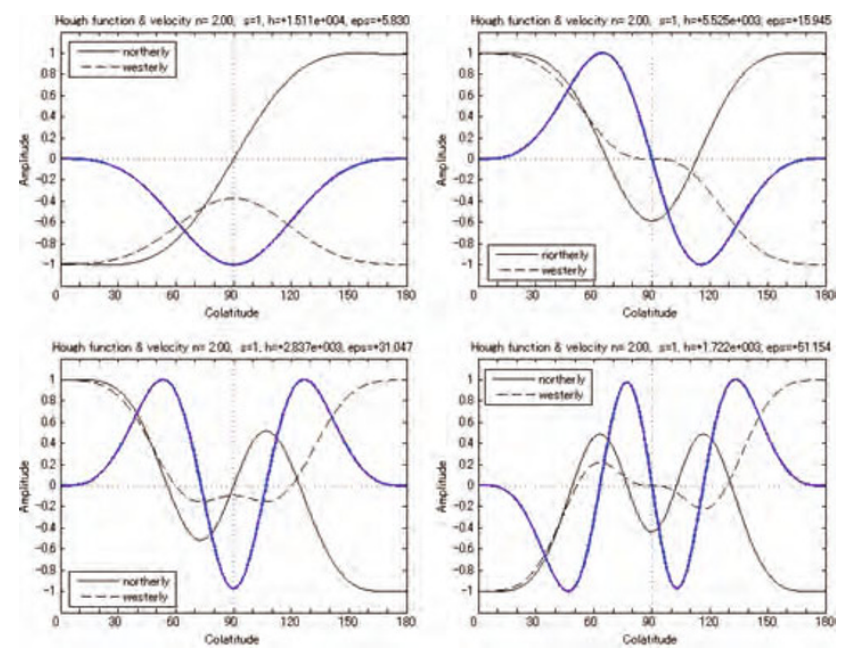

Fig. 1. Hough functions (bold solid line) and velocity expansion functions (solid line for northerly and dashed line for westerly velocity) for semi-diurnal non-migrating tidal modes $(2,1,1),(2,1,2),(2,1,3)$ and $(2,1,4)$

\section{Semidiurnal Non-Migrating Tide at Polar Lat- itudes}

Classical Hough functions and associated velocity expansion functions of the zonal wavenumber $s=1$ westward moving non-migrating semidiurnal tide are shown in Fig. 1. Equivalent depths are $15 \mathrm{~km}$ for the $(2,1,1)$ mode and $5.5 \mathrm{~km}$ for the $(2,1,2)$ mode, which are evanescent and occasionally have a fairly long vertical wavelength. Also, higher modes are $(2,1,3)$ for $2.8 \mathrm{~km}$ and $(2,1,4)$ for $1.7 \mathrm{~km}$ which, for example, have a shorter vertical wavelength of 63 and $44 \mathrm{~km}$, respectively, in the isothermal atmosphere with a temperature of $220 \mathrm{~K}$. Figure 1 indicates that the non-migrating $s=1$ component tends to dominate at polar latitudes and non-zero wind amplitude at the poles. To predict actual behaviors of non-migrating semidiurnal tide at polar latitudes, use is made of the ATM2 modeling (Aso et al., 1987). This linearized steady model solves the coupled partial differential equations of the primitive equation system for tide and planetary waves in the assumed background mean zonal wind and latitudinal temperature gradient, and was coded around the same period as the GSWM developed by Forbes (1982a). Background zonal wind model calculated from CIRA temperature data at June solstice assumes a strong westerly wind maximizing to about $80 \mathrm{~m} / \mathrm{s}$ around $60 \mathrm{~km}$ in the southern winter mid-latitudes and a weaker easterly wind in the northern summer middle atmosphere. In the present modeling, we assumed a simple perpetual heating source of zonal wavenumber $s=1$ component between the upper stratosphere to lower mesosphere heights in the winter hemisphere with height of maximum at $56 \mathrm{~km}$ and half-width of $20 \mathrm{~km}$ as shown in Fig. 2. This forcing is based on the assumption that the non-linear interaction takes place in the winter stratospheric region. As the forcing is assumed in the winter hemisphere and asymmetric in latitude, it is anticipated that anti-symmetric modes contribute to the modeled tidal field. The calculated result for the semidiurnal zonal (westerly) wind component is shown in Fig. 3(a) as a latitude-height 3D surface plot of the am-

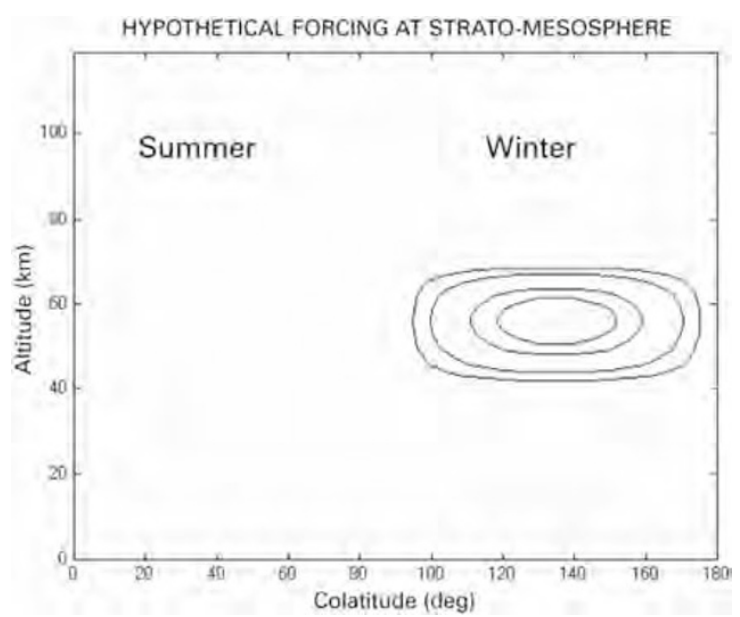

Fig. 2. A latitude-height plot of hypothetical (perpetual) heating with zonal wavenumber one in the winter hemisphere used in the ATM2 modeling for solstice mean wind conditions.

plitude and contour plot of its phase. Amplitude is in linear scale, and it is relative in that the forcing is in itself hypothetical. It is clearly shown that the amplitude indicates enhancement at meteor heights in the summer hemisphere. From phase structures, traveling from winter mesosphere up to summer lower thermosphere in the opposite hemisphere can be traced as is likewise stated in Yamashita et al. (2002). Figure 3(b) shows the temperature component as well. It also tends to enhance in the summer hemisphere with some signature of symmetric $(2,1,3)$ mode suggested, as for the westerly wind component. This mode might be generated through mode coupling by mean zonal wind even in this asymmetric forcing. Figure 4(a) and (b) indicate latitudinal plots of amplitude and phase of northerly (left) and westerly wind components at 80,90 and $100 \mathrm{~km}$ for migrating $s=2$ semidiurnal tide excited by realistic forcing and the present non-migrating $s=1$ semidiurnal tide, respectively, calculated by ATM 2 model. In the figure, amplitude at each height is normalized to unity at its maximum. For migrating semidiurnal tide, amplitudes are definitely larger in the winter hemisphere at 90 and $100 \mathrm{~km}$, and the antisymmetric $(2,3)$ mode is suggested to prevail. Hence, the non-migrating component reveals itself more in summer if it exists. From Fig. 4(a) and (b), it follows that there is some polar latitude where $s=2$ and $s=1$ semidiurnal tides change to dominate in amplitude and poleward of which is dominated by $s=1$ non-migrating components. Either an analysis of available GCM results or, in our model, a quantitative estimate of equivalent forcing emanating from non-linear wave coupling is needed for this purpose. Provisional wavenumber analysis by using two circumpolar stations in the northern hemisphere cannot clearly reveal which of wavenumbers $s=1$ or $s=2$ dominates at these latitudes.

\section{Stationary Planetary Waves and N-S Asymme- try}

Stationary planetary waves are basically very long-period (say 30-40 days) global scale waves and are excited by topography and sensible heat source at lower heights. A 

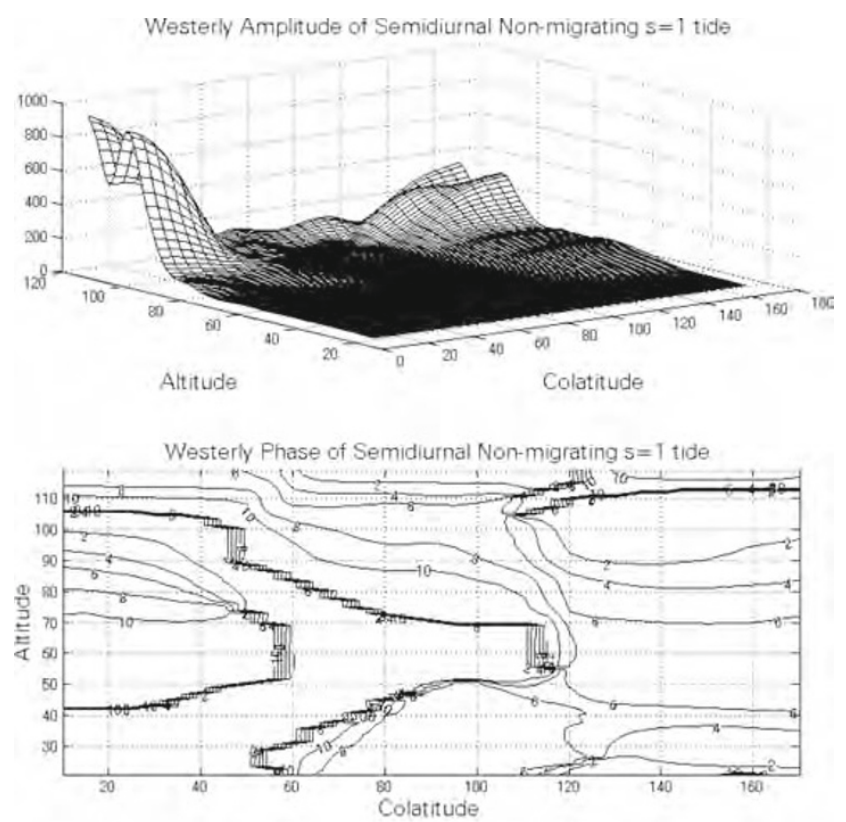
moll 714 surfins 3

$2005420 \quad 16 \quad 58$

(a)

Temperature Amplitude of Semidiurnal Non-migrating $s=1$ tide
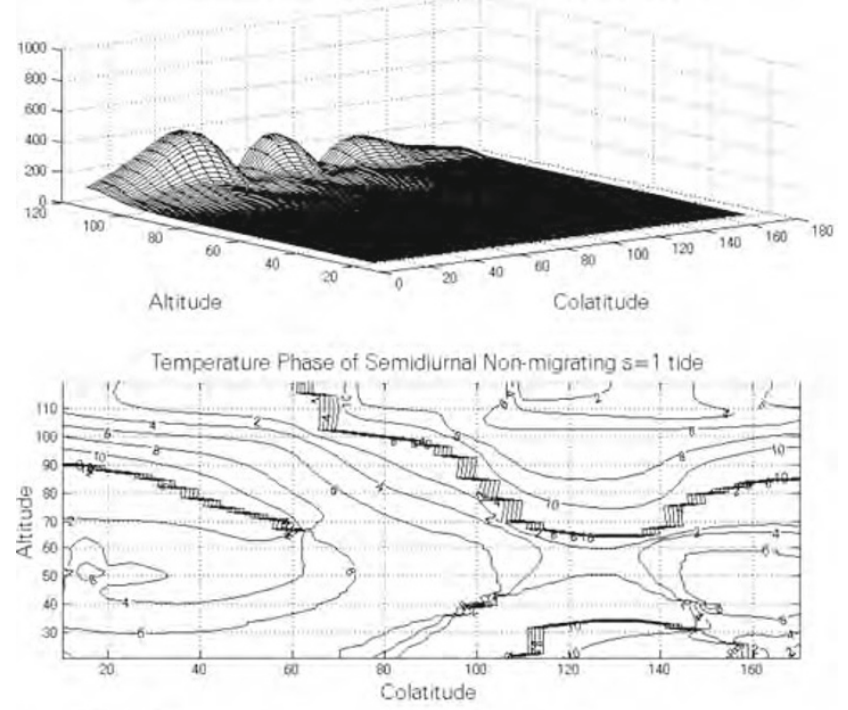

moht7 soutin $3 . \mathrm{m}$

(b)

Fig. 3. A 3D plot of amplitude and a contour plot of phase of the $s=1$ non-migrating semidiurnal (a) westerly wind, and (b) temperature component by the ATM2 modeling excited by perpetual forcing in the winter hemisphere shown in Fig. 2. Solstitial mean zonal wind and temperature are assumed. Amplitude is in linear arbitrary scale.

linearized steady modeling based on the inviscid model (ATM1, Aso et al., 1981), which solves the perturbation geopotential height $p^{\prime} / \rho_{\mathrm{o}}$ ( $p^{\prime}$ : pressure perturbation, $\rho_{\mathrm{o}}$ : atmospheric density), was made by Lindzen et al. (1982) to find out its seasonal variability due to background mean zonal wind. It is generally known that the planetary wave is predominant in the winter hemisphere, and in this calculation its response to topographic forcing tends to be less sensitive to changes in static stability or background wind, in contrast to thermal forcing.

With regard to the activity of the stationary planetaryscale wave of zonal wavenumber $s=1$ in the lower strato-
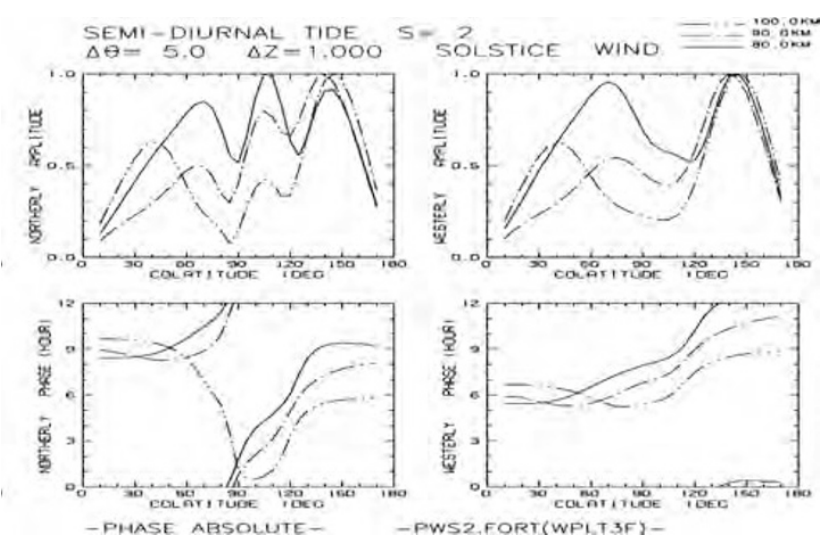

(a)

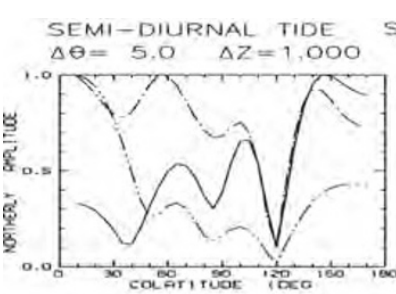

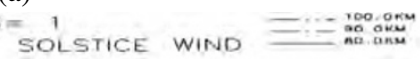
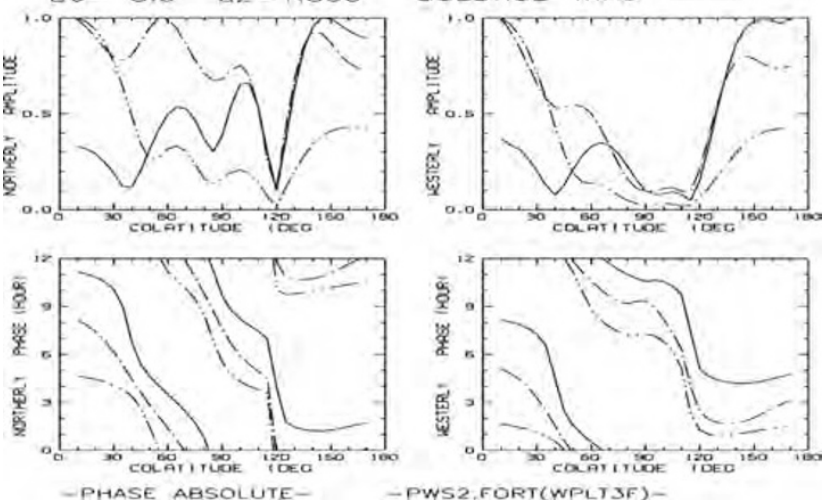

(b)

Fig. 4. Latitudinal plots of amplitude and phase of northerly (left) and westerly wind components for (a) migrating and (b) non-migrating semidiurnal tide at 80,90 and $100 \mathrm{~km}$ calculated by ATM2 model.

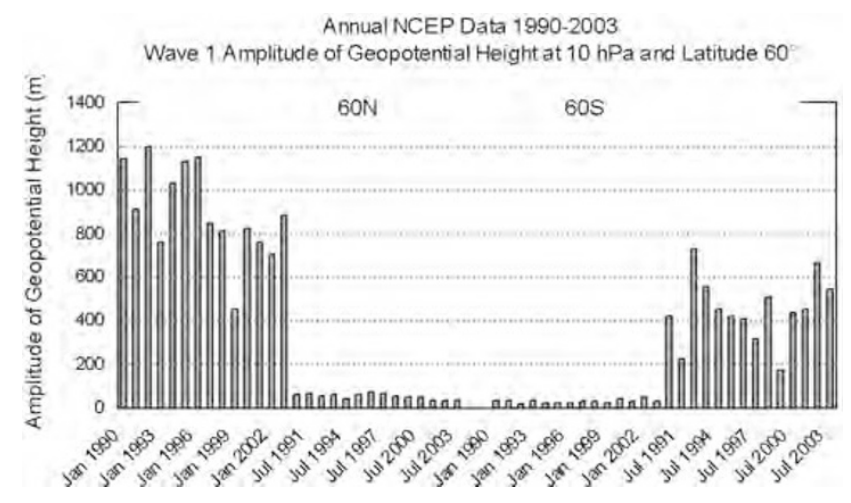

Fig. 5. Comparison of wavenumber $s=1$ amplitude of geopotential height at $10 \mathrm{hPa}$ and latitudes of $60^{\circ}$ at solstices during 1993-2003 between two hemispheres. The data is from the web site of the NCEP/NCAR Reanalysis Project.

sphere, asymmetry is shown to exist between two hemispheres (CIRA:1986, 1990). Here available synoptic meteorological data is investigated to see whether there might be climatological asymmetry in the activity of stationary planetary waves between two hemispheres. Figure 5 compares monthly mean wavenumber 1 amplitude of geopotential height at $10 \mathrm{hPa}$ (ca. $30 \mathrm{~km}$ ) and latitude of $60^{\circ}$ at solstice between two hemispheres based on the NCEP reanalysis data provided by the NOAA-CIRES Climate Diagnostics Center, Boulder, Colorado, USA. These are the 
results obtained between 1990 and 2003. It is very clearly indicated that the northern hemisphere winter time exhibits a larger amplitude than that in the southern hemisphere by a factor of about 2. It is likely that this is due to the surface topography being less evident in the southern hemisphere than in the northern hemisphere. Hence, asymmetry in the zonal wavenumber $s=1$ semidiurnal tide in summer between northern and southern hemispheres, if it exists, very unambiguously supports a suggested scenario by Forbes et al. (1995) and Yamashita et al. (2002) that the zonal wavenumber $s=1$ semidiurnal tide prevalent more in the Antarctic than in the Arctic summer at meteor heights is due to the interaction of the migrating semidiurnal tide and stationary planetary waves of wavenumber $s=1$ which prevails in winter northern hemisphere.

\section{Discussion and Concluding Remarks}

In this note, it is numerically confirmed in a straightforward way by using the ATM2 tidal modeling that the non-migrating semidiurnal tide is excited in the winter stratospheric region and gets through to the opposite summer hemisphere along the weak wind-line. Actually strong westerly wind exceeding $80 \mathrm{~m} / \mathrm{sec}$ around winter mid-latitude increases Doppler shifted frequency by $s V / 2 \Omega a \sin \theta$ ( $V$ : background mean zonal wind, $\Omega$ : earth rotation rate, $a$ : earth radius, $\theta$ : co-latitude) of about $10 \%$, giving rise to longer equivalent depth and a more evanescent characteristic for excited symmetric $(2,1,1)$ or antisymmetric $(2,1,2)$ modes. As shown in Fig. 3(b), temperature component of $s=1$ non-migrating tide shows three humps in the latitudinal plot. Hough mode expansion analysis of this temperature structure reveals that symmetric $(2,1,3)$ modes still dominate along with significant antisymmetric $(2,1,2)$ and $(2,1,4)$ modes with relative magnitudes of 0.3 and 0.5 , respectively. Here we found that the NCEP re-analysis data at $10 \mathrm{hPa}$ shows a climatological disparity of $s=1$ geopotential height activity between $60^{\circ} \mathrm{N}$ and $60^{\circ} \mathrm{S}$, the former being larger than the latter for most of the year. It is then postulated that the asymmetry of the nonmigrating semidiurnal tide can be caused by the difference in planetary wave activity in the winter polar stratospheric region and concomittant propagation into the opposite summer hemisphere meteor heights.

We suggest that there might be sensible asymmetry in summertime enhancement of semidiurnal tide between both polar regions. A quick-look review of the northern highlatitude $\left(78^{\circ} \mathrm{N}\right)$ meteor radar observations indicate that the predominance of semidiurnal oscillation in summer is no more well defined than in other seasons, although migrat- ing and non-migrating components are not resolved therein. Also, amplitudes observed at $78^{\circ} \mathrm{N}$ intermittently exceed those observed at $70^{\circ} \mathrm{N}$, mostly during equinoctial to early solstitial months, and hence existence of the non-migrating component in summertime is not significantly suggested in terms of latitudinal amplitude excursion. Details on these will be published elsewhere (Aso, Tsutsumi and Hall, in preparation, 2007) but the results seem to be in contrast to reported enhancement in the conjugate South Pole region. This asymmetry is not quite conclusive, and comprehensive bipolar studies are crucial in focusing on circumpolar zonal wave-numbers by collaborative radars and optics which deploy in latitude and longitude in the Arctic and Antarctic regions.

Acknowledgments. NCEP re-analysis data is provided by the NOAA-CIRES Climate Diagnostics Center, Boulder, Colorado, USA, from their Web site at http://www.cdc.noaa.gov/

\section{References}

Aso, T., T. Nonoyama, and S. Kato, Numerical simulation of semidiurnal atmospheric tides, J. Geophys. Res., 86(A13), 11388-11400, 1981.

Aso, T., S. Ito, and S. Kato, Background wind effect on the diurnal tide in the middle atmosphere, J. Geomag. Geoelectr., 39, 297-3005, 1987.

CIRA:1986, COSPAR International Reference Atmosphere, Part 2, Middle atmosphere models, Adv. Space Res., 10(12), edited by D. Rees et al., 517pp, Pergamon Press, 1990.

Forbes, J. M., Atmospheric Tides. I. Model Description and Results for the Solar Diurnal Component, J. Geophys. Res., 87, 5222-5240, 1982a.

Forbes, J. M., Atmospheric Tides. II. The Solar and Lunar Semidiurnal Components, J. Geophys. Res., 87, 5241-5252, 1982b.

Forbes, J. M., N. A. Makarov, and Y. I. Portnyagin, First results from the meteor radar at South Pole: a large 12-hour oscillation with zonal wavenumber one, Geophys. Res. Lett., 22(23), 3247-3250, 1995.

Forbes, J. M., Y. I. Portnyagin, N. A. Makarov, S. E. Palo, E. G. Merzlyakov, and X. Zhang, Dynamics of the lower thermosphere over South Pole from meteor radar wind measurements, Earth Planets Space, 51, 611-620, 1999.

Lindzen, R. S., T. Aso, and D. Jacqmin, Linearized calculations of stationary waves in the atmosphere, J. Meteor. Soc. Japan, 60(1), 66-77, 1982.

Miyahara, S., Y. Miyoshi, and K. Yamashita, Variations of migrating and non-migrating tides simulated by the middle atmosphere circulation model at Kyushu University, Adv. Space. Res., 24(11), 1549-1558, 1999

Portnyagin, Yu. I., J. M. Forbes, N. A. Makarov, E. G. Merzlyakov, and S. Palo, The summertime 12-hour wind oscillation with zonal wavenumber $s=1$ in the lower thermosphere over the South Pole, Ann. Geophys., 16, 828-837, 1998.

Yamashita, K., S. Miyahara, Y. Miyoshi, K. Kawano, and J. Ninomiya, Seasonal variation of non-migrating semidiurnal tide in the polar MLT region in a general circulation model, J. Atmos. Solar-Terr. Phys., 64, 1083-1094, 2002.

T. Aso (e-mail: t-aso@nipr.ac.jp) 